\title{
MOLECULAR DYNAMICS SIMULATIONS OF AN ANOMALOUS RESPONSE OF DIAMOND TO SHOCK COMPRESSION
}

\author{
K. McLaughlin ${ }^{1}$, I.I. Oleynik ${ }^{1}$, S.V. Zybin ${ }^{2}$, M. L. Elert $^{3}$, and C. T. White ${ }^{4}$ \\ ${ }^{1}$ Department of Physics, University of South Florida, Tampa, FL 33620 \\ ${ }^{2}$ Materials and Process Simulation Center, California Institute of Technology, Pasadena, CA 91125 \\ ${ }^{3}$ Chemistry Department, U. S. Naval Academy, Annapolis, MD 2140 \\ ${ }^{4}$ Naval Research Laboratory, Washington, DC 20375
}

\begin{abstract}
We performed molecular dynamics simulations of shock wave propagation in diamond in the [110] crystallographic direction and observed an anomalous response of the material. This regime is characterized by absence of plastic deformation in the intermediate interval of shock wave intensities between shear-deformation and overdriven rehybridization shock wave regimes.
\end{abstract}

Keywords: Shock waves, shear stresses, molecular dynamics, diamond

PACS: $62.50 .+\mathrm{p}, 82.40 . \mathrm{Fp}, 81.30 . \mathrm{Hd}, 46.40 . \mathrm{Cd}$

\section{INTRODUCTION}

Shock compression of condensed matter is a vital tool in the investigation of the fundamental properties of solids at high pressures and temperatures. Such conditions are realized in geothermal processes, hyper-velocity impacts and detonation of energetic materials. However, due to the sub-nanometer, sub-picosecond length and time scales involved, obtaining atomic-scale information remains an experimental challenge. In contrast, these are precisely the scales in which atomistic simulation becomes invaluable [1-3]. Molecular dynamics (MD) simulations of millions of atoms over a picosecond time scale may yield valuable information on shock velocities, thermodynamic variables and the atomic scale structure of the shock front, while also providing valuable insight concerning plastic deformation, phase transitions and materials defects.

Although much focus has been applied to determining the properties of shock compressed metals [4-5], interest in other classes of materials has been on the rise, such as with covalently bonded solids and ceramics [6-8]. Diamond, in particular, is striking due to its phenomenal mechanical properties, having the highest bulk modulus of all elemental solids and superb resistance to shear deformations [9]. Experiments have provided some insight into the material transformations of diamond under dynamical loading, but the atomic-scale mechanisms are not well understood.

We present results of MD simulations of shockwave propagation in diamond single crystals along the [110] crystallographic direction. The MD simulations reveal the existence of an anomalous response at the intermediate interval of shock wave intensities between shear-deformation and overdriven rehybridization shock waves regimes. Our major goal is to investigate the mechanisms that are responsible for this anomalous response and their relationship to the underlying constitutive materials properties which result in this unforeseen behavior. 


\section{COMPUTATIONAL DETAILS}

Our MD simulations were performed using the reactive empirical bond-order (REBO) interatomic potential [10-11] which is widely considered as one of the best currently available potentials for carbon. Initially a single crystal sample, oriented in the [110] crystallographic direction is given an initial velocity $-u_{p}$ in the $z$-direction with $u_{p}$ ranging from 1.0 to $7.2 \mathrm{~km} / \mathrm{s}$ with increments of $0.2 \mathrm{~km} / \mathrm{s}$. Periodic boundary conditions are applied in the $\mathrm{x}$ and $y$ directions of this crystal, which consists of approximately 3.4 million atoms and is $200-300$ angstroms in each dimension.

As the crystal collides with a piston of infinite mass, represented by a repulsive potential barrier, as many as two shock waves propagate along the $\mathrm{z}$ axis. These elastic and shear-deformation shock waves may be characterized by substantial changes in the local variables, such as temperature, stress, mass velocity, density, and local potential energy, which are calculated using spatial averaging over the direction of shock propagation.

\section{RESULTS AND DISCUSSION}

The simulations of shock wave propagation revealed four regimes of material response. They are: (a) a pure elastic shock wave at piston velocities $\mathrm{u}_{\mathrm{p}}<1.8 \mathrm{~km} / \mathrm{s}$ (b) a split shock wave

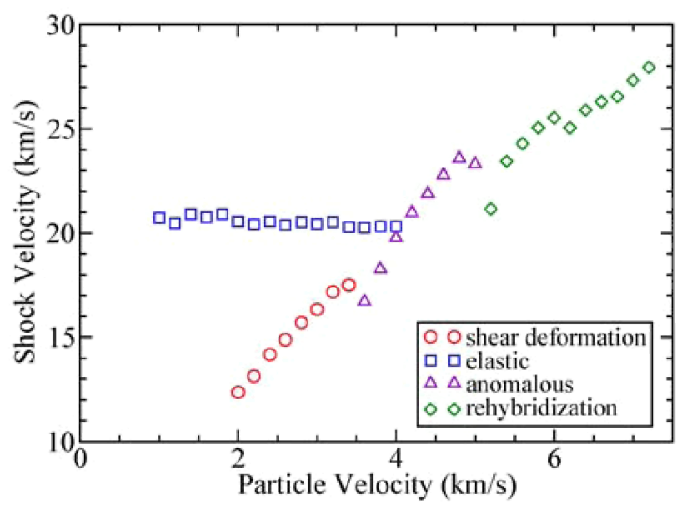

FIGURE 1. The shock Hugoniot for diamond [110].

consisting of an elastic precursor followed by a slower shear-deformation shock wave at $2.0 \mathrm{~km} / \mathrm{s}<$ $\mathrm{u}_{\mathrm{p}}<3.4 \mathrm{~km} / \mathrm{s}$; (c) an anomalous shock wave at 3.6 $\mathrm{km} / \mathrm{s}<\mathrm{u}_{\mathrm{p}}<5.0 \mathrm{~km} / \mathrm{s}$; and finally (d) an overdriven rehybridization shock wave at the highest shock intensities, $u_{p}>5.2 \mathrm{~km} / \mathrm{s}$. These different regimes of materials response are conveniently classified using the shock Hugoniot, which gives the dependence of shock velocity on the piston velocity, see Figure 1.

The pure elastic regime is observed in simulations with piston velocities between 1.0 and $1.6 \mathrm{~km} / \mathrm{s}$, with a constant shock wave velocity of about $20.3 \mathrm{~km} / \mathrm{s}$. This elastic shock wave is best described as a strictly uniaxial compression with no

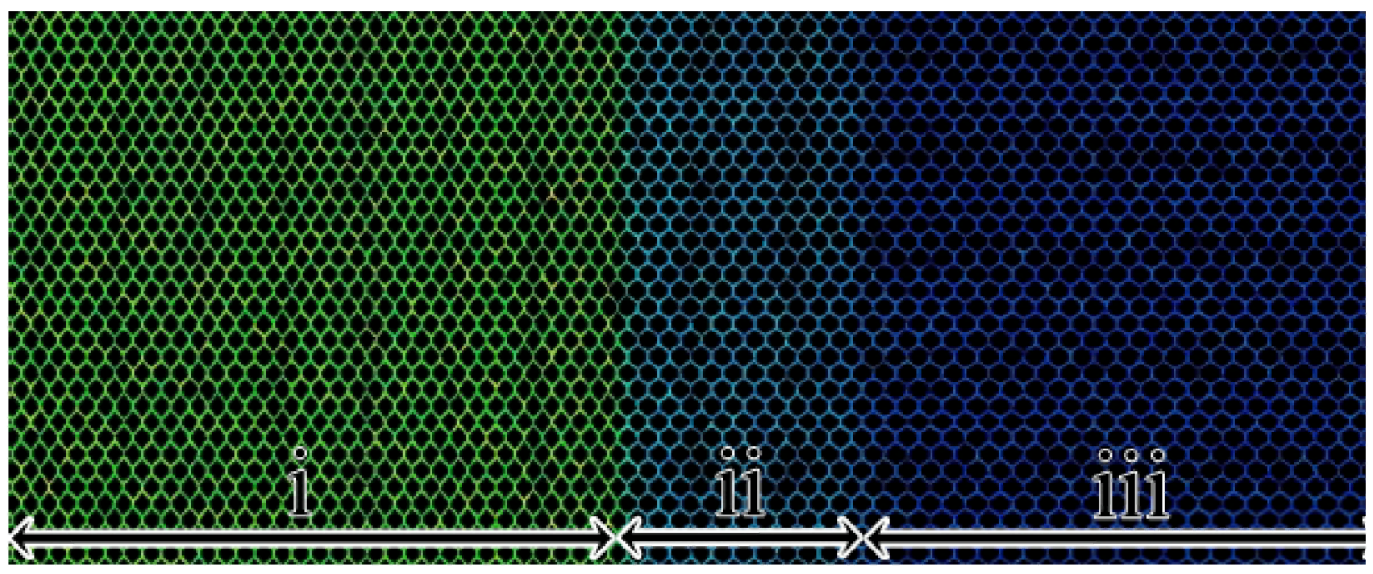

FIGURE 2. Two shock waves propagating through diamond [110] with particle velocity $\mathrm{u}_{\mathrm{p}}=3.8 \mathrm{~km} / \mathrm{s}$. The three regions above are identified as follows: i) anomalous, ii) elastic and iii) unperturbed diamond crystal. 

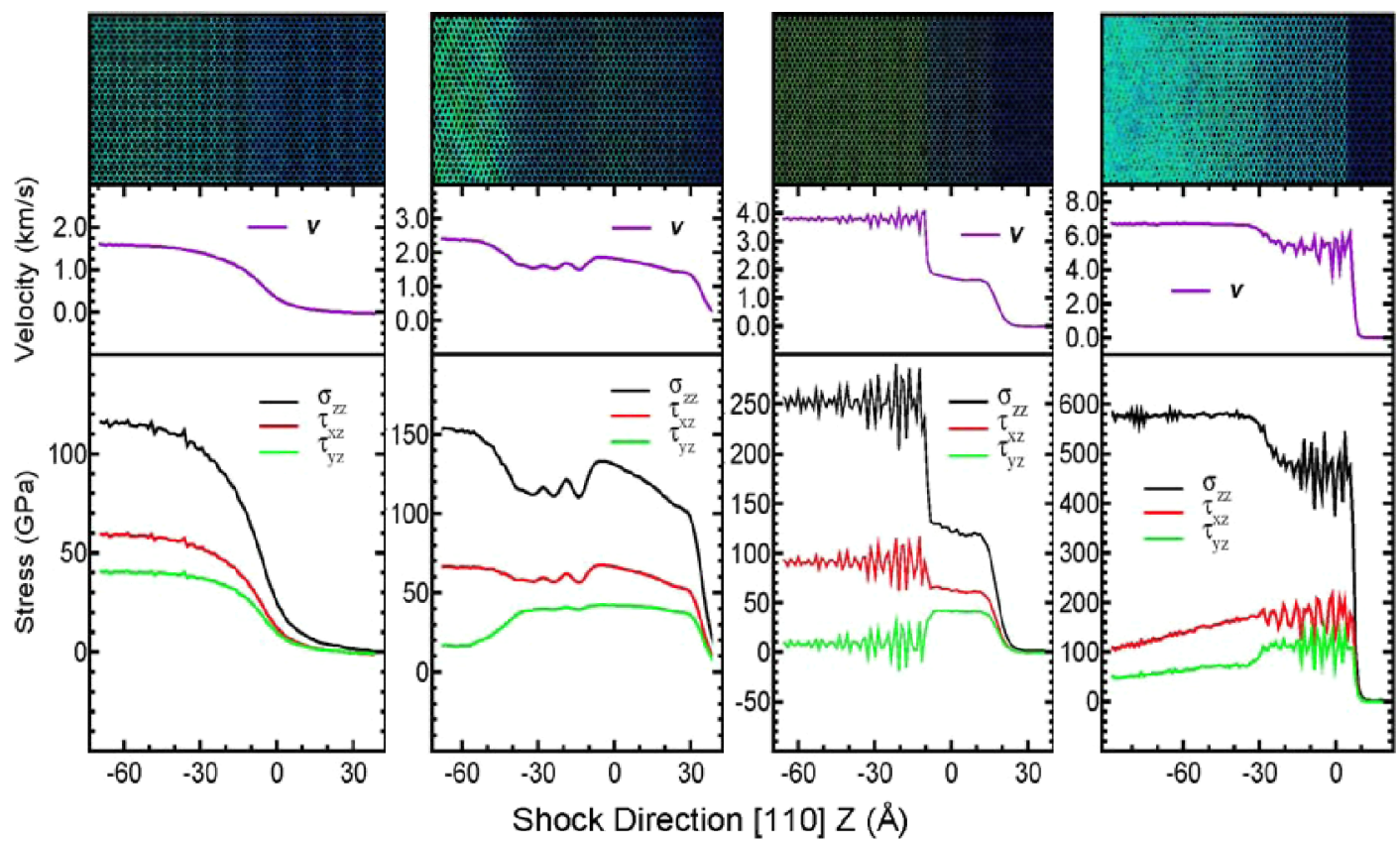

FIGURE 3. Shock wave profiles for the four regimes present in diamond [110], $\mathrm{u}_{\mathrm{p}}=2.0$ to $7.2 \mathrm{~km} / \mathrm{s}$. From left to right: elastic, plastic, anomalous, and rehybridization.

evidence of changes in crystal structure, see Figure 3. For each of these cases, a smooth increase in the local variables is seen with a maximum longitudinal stress $\sigma_{\mathrm{zz}}$ of approximately $120 \mathrm{GPa}$, and shear stresses $\tau_{\mathrm{xz}}<60 \mathrm{GPa}$ and $\tau_{\mathrm{yz}}<40 \mathrm{GPa}$

By increasing the particle velocity to values between 1.8 and $3.4 \mathrm{~km} / \mathrm{s}$, the critical shear stress values are reached, and a shear-deformation shock wave appears, which propagates with the velocity $\mathrm{u}_{\text {shear }}, 12.4 \mathrm{~km} / \mathrm{s}<\mathrm{u}_{\text {shear }}<17.5 \mathrm{~km} / \mathrm{s}$, along with an elastic precursor, which propagates with the velocity $u_{\text {elastic }}=20.3 \mathrm{~km} / \mathrm{s}$. Although in our simulations the time scales are insufficient to observe irreversible plastic deformations in this regime, it is believed that such plasticity, in fact, will be developed in the course of attaining an equilibrium state far behind the shock wave front.

The local variables behind the elastic shock front remain constant, and equal to the values observed at the critical particle velocity $\mathrm{u}_{\mathrm{p}}=1.8$ $\mathrm{km} / \mathrm{s}$, see Figure 3 . Behind the shear deformation wave, a decrease of shear stress $\tau_{\mathrm{yz}}$ is observed, with the value dropping from 40 to $15 \mathrm{GPa}$ in the $u_{p}=2.0 \mathrm{~km} / \mathrm{s}$ case. Clearly, these deformations are due to the relaxation of the crystal into a lower energy state. The shear deformation wave is further characterized by a large increase in temperature with values reaching $500 \mathrm{~K}$, as compared to $350 \mathrm{~K}$ behind elastic fronts.

For $\mathrm{u}_{\mathrm{p}}=3.6$ to $5.0 \mathrm{~km} / \mathrm{s}$ an anomalous shock wave, $16.7 \mathrm{~km} / \mathrm{s}<\mathrm{u}_{\text {anomalous }}<23.3 \mathrm{~km} / \mathrm{s}$, is present. At the lower end of this range, the anomalous shock wave is coupled with an elastic precursor, while only an overdriven anomalous wave is present when $u_{p}>4.0 \mathrm{~km} / \mathrm{s}$. The key feature of this response is a complete disappearance of the shear deformations that characterize the second regime, see Figure 2.

In terms of local thermodynamic quantities, the anomalous shock wave is characterized by a large peak followed by rapid fluctuations immediately behind the shock front, see Figure 3. Within this regime there is a large increase in temperature which peaks at $\mathrm{T}_{\mathrm{yy}}=1300 \mathrm{~K}, \mathrm{~T}_{\mathrm{zz}}=1000 \mathrm{~K}$, before 
thermal equilibrium is attainted at about $\mathrm{T}_{\mathrm{xx}}=\mathrm{T}_{\mathrm{yy}}=$ $\mathrm{T}_{\mathrm{zz}}=800 \mathrm{~K}$. In addition, the anomalous front is also accompanied by a decrease in $\tau_{\mathrm{yz}}$ from 41 to -3 $\mathrm{GPa}$, while $\tau_{\mathrm{xz}}$ and $\sigma_{\mathrm{zz}}$ increase from 66 and 127 $\mathrm{GPa}$ to 80 and $230 \mathrm{GPa}$, respectively.

Finally, the overdriven rehybridization regime is realized when $u_{p}>5.0 \mathrm{~km} / \mathrm{s}$. Initially, the features of this shock wave are identical to those of the anomalous waves, with a sudden peak in local variables, $\tau_{\mathrm{xz}}=200 \mathrm{GPa}, \tau_{\mathrm{yz}}=140 \mathrm{GPa}, \rho=4.5$ $\mathrm{g} / \mathrm{cm}^{3}$, followed by fluctuations, see Figure 3 . However, in this case, the large shock wave intensity is inevitably transformed into chaotic atomic motion within the crystal lattice leading to a large increase in temperature. Ultimately, these temperatures rise to a point where localized regions of graphitization and melting begin to appear, and consequently, all of the shear stresses steadily drop to zero.

Taken together, our results suggest that the Hugoniot elastic limit, that is, the critical value of a shock wave intensity resulting in onset of plastic deformations, is not solely related to the longitudinal stress. On the contrary, as seen with the disappearance of shear-deformations and the onset of the anomalous response at intermediate shock intensities, the critical shear stresses should be considered as well. Moreover, we believe this anomalous response has a direct link to the nonmonotonic relationship of these shears with respect to uniaxial strain.

Although the topic of the Hugoniot elastic limit, as it relates to these results, will be covered in more detail elsewhere [12], we believe that these results reveal a need to generalize the notion of the Hugoniot elastic limit in such a way as to include critical shear stresses.

\section{ACKNOWLEDGEMENTS}

The work at USF was supported by the Office of Naval Research (ONR) through the Naval Research Laboratory (NRL). The work at NRL was also supported by ONR both directly and through NRL. K. McLaughlin thanks the NSF IGERT Program Grant DGE 0221681 (Bernard Batson, the manager), USF Office of Undergraduate Research, and the organizing committee of SCCM 2007 (Ricky Chau) for the travel support to attend
SCCM 2007 in Hawaii.

\section{REFERENCES}

1. B.L. Holian, Shock Waves 13, 489 (2004).

2. S.V. Zybin, V.V. Zhakhovskii, M.L. Elert, and C.T. White, in Shock Compression of Condensed Matter - 2003, edited by M.D. Furnish and Y.M. Gupta (AIP Press, NY, 2004), pp. 310-313.

3. D.H. Robertson, D.W. Brenner, and C.T. White, Phys. Rev. Lett. 67, 3132 (1991),

4. K. Kadau, T.C. Germann, P.S. Lomdahl, and B.L. Holian, Science 296, 1681 (2002)

5. K. Kadau, T.C. Germann, P.S. Lomdahl, and B.L. Holian, Phys. Rev. B 72, 064120 (2005).

6. T. Mashimo, R. Chau, Y. Zhang, T. Kobayoshi, T. Sekine, K. Fukuoka, Y. Syono, M. Kodama, and W.J. Nellis, Phys. Rev. Lett. 96, 105504 (2006).

7. M. Chen, J.W. McCauley, and K.J. Hemker, Science 299, 1563 (2003).

8. M.W. Chen, J.W. McCauley, D.P. Dandekar, and N.K. Bourne, Nature Materials 2006, 814 (2006).

9. Properties of Natural and Synthetic Diamond, edited by J.E. Field (Academic Press, 1992).

10. D.W. Brenner, Phys. Rev. B 42, 9458 (1990).

11. D.W. Brenner, O.A. Shenderova, J.A. Harrison, S.J. Stuart, B. Ni and S.B. Sinnott, J. Phys. Cond. Matt. 14, $783(2002)$.

12. I.I Oleynik, K. McLaughlin, S.V. Zybin, M. Elert, and C.T. White, to be published. 